\title{
Decellularized scaffolds for neuronal regeneration
}

\author{
Raffa $\mathrm{P}^{1,2}$ and Urciuolo $\mathrm{A}^{1,3 *}$ \\ ${ }^{1}$ Women's and Children's Health Department, University of Padova, Italy \\ ${ }^{2}$ Venetian Institute of Molecular Medicine, Padova, Italy \\ ${ }^{3}$ Institute of Pediatric Research IRP, Padova, Italy
}

The extracellular matrix (ECM) provides the three-dimensional structure of tissues, and is required for cell homing and cell viability, as well as for the overall homeostasis of tissues and organs [1,2]. The dynamic and complex microenvironment that the ECM generates in a specific manner for each tissue guarantees its functions [1,2]. During tissue regeneration ECM has been shown to play an essential role in controlling the tissue-stem cell compartment and to be involved in tissue regeneration outcome [3-6]. Tissue engineering combines extracellular natural and/or synthetic scaffolds (biomaterials) with stem cells and growth factors for the development of regenerative medicine strategies and the treatment of diseased tissues [7]. Despite the fact that incredible improvements have been achieved in biomaterial manufacturing, the peculiar and complex biochemistry, biomechanics and 3D organization proper of a tissue-specific ECM still cannot totally be reproduced in the lab $[1,2,8]$. Such complexity can however be preserved in scaffolds that take advantage of the native tissue themselves, as decellularized tissues or whole organs [9-11]. Decellularization process remove cellular and nuclear content retaining ECM mechanical integrity, biological activity and 3D architecture of the native tissue [10]. Decellularized tissues and/or organs represent alternative and promising scaffold material for the treatment of clinical cases in which extensive regeneration of an organ is required, as in cases of traumatic injuries, surgical ablation and congenital diseases [12]. Decellularized scaffolds have already been obtained from different organs and used for regenerative medicine strategies in animal models, as well as in clinical trials $[12,13]$. Ideally scaffold implantation should allow regeneration of the tissue of interest and guarantee the functionality of the targeted organ. In particular, support in reinnervation is a crucial aspect for the final outcome of tissue functionality in those organs in which the nervous system plays pivotal roles (i.e. muscle, heart, sensory organs). Here we will summarize the state of the art regarding the possible use of decellularized scaffolds for spinal cord and peripheral nervous system (PNS) regeneration, and tissue reinnervation.

Despite the ability of PNS to maintain a grade of regeneration after injury, central nervous system (CNS) axons do not regenerate appreciably in their native environment [14-16]. In PNS, after a nerve transection, macrophages and Schwann cells clear myelin and axonal debris, and produce cytokines that enhance axon growth $[17,18]$. After debris clearance, regeneration begins at the proximal end of the damage and continues toward the distal stump with a section of new tissue known as 'the bridge' and is composed of inflammatory cells, perineurial cells, fibroblasts and ECM $[14,18]$. For the complete functional reinnervation of the organs, axons have to extend until they reach their distal target [15]. This requires the formation of cellular cords of Schwann cells (dedifferentiated to progenitor-like cells) and fibroblasts, which transport the axons across the bridge along the surface of polarised blood vessels [14]. Differently, when CNS undergoes injury the regeneration capability is inhibited by the bloodspine barrier, which reduce the infiltration of macrophages at the site of injury, delaying the removal of inhibitory myelin and resulting in a glial scar formation [15]. However, in case of a severe injury the regeneration of spinal cord, or PNS or tissue innervation fails $[15,16]$.

To develop new strategies for spinal cord and PNS regeneration, natural or artificial synthetic materials have been extensively used [19-24], and spinal cord biocompatible decellularized scaffolds were first obtained in 2010 [25]. Liu and colleagues provided evidence that decellularized spinal cord scaffolds seeded with human umbilical cord blood-derived mesenchymal stem cells (hUCB-MSCs) are able to bridge a spinal cord cavity and promote long-distance axon regeneration and functional recovery in a spinal cord injury (SCI) rat model [26]. This study supports the hypothesis that decellularized spinal cord can provide a favourable environment for host oligodendrocyte differentiation, proliferation and axonal remyelination, which promotes endogenous regeneration of neural cells [26]. However, the influence of hUCB-MSCs, which secrete a number of cytokines useful for SCI recovery [27], in the general outcome of the regeneration was not clear. More recently, the ability of decellularized spinal cord to promote regeneration per se (without pre-seeding cells) has been demonstrated $[28,29]$. Scaffolds provided the right topography and direction for nerve axon regeneration of removed T9-T10 cord segments in rats, with improvement in locomotor function compared to the untreated SCI group [28]. Xu and collaborators also showed how decellularized spinal cord can be used in combination with trophic factors to improve the regenerative properties of the implanted matrix [29]. Remarkable results in terms of functional recovery in SCI models have been obtained also using injectable decellularized extracellular matrix derived from brain [30], meninges [31] or peripheral nerve [32].

Decellularized nerves have also been used in animal models [33] and for clinical application [34]. In sciatic nerve replacement rat models, decellularized nerves promoted axonal regeneration and motor function recovery [35]. Interestingly, bone marrow-mesenchymal stromal cells (BM-MSCs) embedded in fibrin glue and injected around the graft helped to improve nerve regeneration and functional recovery [35]. Importantly, clinical application of a commercial decellularized nerve graft (AxoGen ${ }^{\oplus}$, AxoGen Inc, Alachua, FL) showed promising results. Patients with digital nerve defect showed no signs of infection

${ }^{\star}$ Correspondence to: Anna Urciuolo, Institute of Pediatric Research IRP, Padova, Italy, E-mail: anna.urciuolo@unipd.it

Received: January 10, 2020; Accepted: January 20, 2020; Published: January 23, 2020 
or rejection after decellularized nerve implantation, and reported sensory improvement in a follow-up study [36].

The ability of decellularized scaffolds to promote in vivo regeneration of neuronal compartment in innervated tissues has been investigated in different organs and by using scaffolds derived from several sources. Decellularized small intestinal submucosa (SIS) scaffolds used to repair esophagus [37] and bladder [38] defects (in rats and canine models respectively) allowed de novo innervation of regenerated implants. Importantly, in the esophagus it was shown that decellularized SIS scaffolds promoted regeneration of synaptic active area, with functional activity of the tissue characterized by contractile responses to nerve stimulation [37]. Typical features of amyelinic fibers being surrounded in groups by single Schwann cells were identified in decellularized heart valves in pig models of valves transplantation [39]. Decellularized cornea scaffolds promoted active reinnervation of the implant by the host cells, as confirmed by the presence of multiple nerve filaments 4 months after transplantation [40].

Considering the essential role of the nervous system in skeletal muscle function, a number of studies investigated with more detail the ability of implanted decellularized scaffolds in supporting axon invasion. We recently showed that two months after implantation in mouse, a decellularized muscle xenograft promoted tissue regeneration in a volumetric muscle loss mouse model, which included neuronal compartment and active neuromuscular junction [41]. In agreement with this, other studies demonstrated that decellularized skeletal muscle scaffolds implanted in vivo are able to restore not only muscle mass, but also muscle innervation and functional recovery [42-44]. In particular, improved maturation of neuromuscular junctions was observed in exercised mice subject to tibialis anterior volumetric muscle loss damage [43]; in a diaphragmatic hernia mouse model, decellularized scaffold was able to guide nerve attraction and re-growth and to direct development of new neuromuscular junctions [44]. Moreover, an interesting work investigated the possibility to recreate ad hoc decellularized scaffold for oriented tissue regeneration [45].

In recent years, remarkable progresses have been reached in the use of biomaterials for promoting neuronal regeneration. Regardless that further studies are required to understand the role of tissue-specific decellularized matrices in neuronal regeneration, decellularized scaffolds still represent promising biomaterials for the development of alternative regenerative strategies [46]. It was recently shown that the combination of removable polymeric microfibers and decellularized matrix allowed the generation of decellularized ECM scaffolds with aligned microchannels able to guide proliferation and differentiation of nervous cells in vitro, and to sustain tissue repair in vivo [45]. The technical integration of synthetic biomaterials and decellularized scaffolds, as whole tissues, as well as injectable gel $[47,48]$ or bio-ink suitable for bioprinting [49,50], represents a powerful instrument to finely manipulate three-dimensional scaffolds and therefore to instruct cell behaviour and improve the overall outcome of tissue regeneration.

\section{Acknowledgments}

This work was supported by STARS Starting Grant 2017 (Grant Code: LS3-19613).

\section{Conflicts of interest}

No competing interests exist.

\section{References}

1. Frantz C, Stewart KM, Weaver VM (2010) The extracellular matrix at a glance. J Cell Sci 123: 4195-4200.

2. Humphrey JD, Dufresne ER, Schwartz MA (2014) Mechanotransduction and extracellular matrix homeostasis. Nat Rev Mol Cell Biol 15: 802-812.

3. Gattazzo F, Urciuolo A, Bonaldo P (2014) Extracellular matrix: A dynamic microenvironment for stem cell niche. Biochimica et Biophysica Acta - General Subjects 1840: 2506-2519.

4. Agren MS, Werthen M (2007) The Extracellular Matrix in Wound Healing: A Closer Look at Therapeutics for Chronic Wounds. Int J Low Extrem Wounds 6: 82-97.

5. Watt FM, Huck WTS (2013) Role of the extracellular matrix in regulating stem cell fate. Nat Rev Mol Cell Biol 14: 467-473.

6. Brown BN, Badylak SF (2014) Extracellular matrix as an inductive scaffold for functional tissue reconstruction. Transl Res 163: 268-285.

7. Atala A, et al. (2018) Principles of regenerative medicine. (Academic Press, 2018).

8. Williams DF (2019) Challenges with the Development of Biomaterials for Sustainable Tissue Engineering. Front Bioeng Biotechnol 7: 1-10.

9. Hillebrandt $\mathrm{KH}$, et al. (2019) Strategies based on organ decellularization and recellularization. Transpl Int tri 32: 571-585.

10. Gilbert TW, Sellaro TL, Badylak SF (2006) Decellularization of tissues and organs. Biomaterials 27: 3675-3683.

11. Urciuolo A, De Coppi P (2018) Decellularized Tissue for Muscle Regeneration. Int. J. Mol. Sci 19: 14808-14831

12. Yu Y, et al. (2016) Decellularized scaffolds in regenerative medicine. Oncotarget 7 : 58671-58683.

13. Taylor DA, Sampaio LC, Ferdous Z, Gobin AS, Taite LJ (2018) Decellularized matrices in regenerative medicine. Acta Biomaterialia 74:74-89.

14. Cattin AL, Lloyd AC (2016) The multicellular complexity of peripheral nerve regeneration. Curr. Opin. Neurobiol 39: 38-46.

15. Schmidt CE, Leach JB (2003) Neural Tissue Engineering: Strategies for Repair and Regeneration. Annu. Rev. Biomed. Eng 5: 293-347.

16. Olson L (1997) Regeneration in the adult central nervous system: Experimental repair strategies. Nat. Med 3: 1329-1335.

17. Lewis GM, Kucenas S (2014) Perineurial Glia Are Essential for Motor Axon Regrowth following Nerve Injury. J. Neurosci. 34: 12762-12777.

18. Cattin AL, et al. (2015) Macrophage-Induced Blood Vessels Guide Schwann CellMediated Regeneration of Peripheral Nerves. Cell 162: 1127-1139.

19. Patist CM, et al. (2004) Freeze-dried poly (d,1-lactic acid) macroporous guidance scaffolds impregnated with brain-derived neurotrophic factor in the transected adult rat thoracic spinal cord. Biomaterials 25: 1569-1582.

20. Novikova LN, et al. (2008) Biodegradable poly- $\beta$-hydroxybutyrate scaffold seeded with Schwann cells to promote spinal cord repair. Biomaterials 29: 1198-1206.

21. Stokols S, et al. (2006) Templated Agarose Scaffolds Support Linear Axonal Regeneration. Tissue Eng 12: 2777-2787.

22. Chen N, et al. (2016) Nanobiomaterials for neural regeneration. Neural Regen. Res 11: 1372-1374.

23. Subramanian A, Krishnan UM, Sethuraman S (2009) Development of biomaterial scaffold for nerve tissue engineering: Biomaterial mediated neural regeneration. $J$ Biomed Sci 16: 108.

24. Teleanu RI, et al. (2019) Nanomaterial-Based Approaches for Neural Regeneration Pharmaceutics 11: 266

25. Guo SZ, et al. (2010) Preparation of the acellular scaffold of the spinal cord and the study of biocompatibility. Spinal Cord 48: 576-581.

26. Liu J, et al. (2013) Acellular spinal cord scaffold seeded with mesenchymal stem cells promotes long-distance axon regeneration and functional recovery in spinal cord injured rats. J. Neurol. Sci. 325: 127-136.

27. Neuhoff S, et al. (2007) Proliferation, differentiation, and cytokine secretion of human umbilical cord blood-derived mononuclear cells in vitro. Exp. Hematol 35: 1119-1131. 
28. Zhu J, et al. (2018) Effect of decellularized spinal scaffolds on spinal axon regeneration in rats. J. Biomed. Mater. Res. Part A 106: 698-705.

29. Xu HL, et al. (2016) Thermo-sensitive hydrogels combined with decellularised matrix deliver bFGF for the functional recovery of rats after a spinal cord injury. Sci. Rep. 6: $1-15$.

30. Hong JY, et al. (2020) Decellularized brain matrix enhances macrophage polarization and functional improvements in rat spinal cord injury. Acta Biomater 101: 357-371.

31. Vishwakarma SK, Bardia A, Lakkireddy C, Basha Paspala SA, Khan AA, et al (2018) Bioengineering human neurological constructs using decellularized meningeal scaffolds for application in spinal cord injury. Front. Bioeng. Biotechnol 6: 1-19.

32. Cornelison RC, et al. (2018) Injectable hydrogels of optimized acellular nerve for injection in the injured spinal cord. Biomed. Mater 13: 034110.

33. Lovati AB, et al. (2018) Nerve Repair Using Decellularized Nerve Grafts in Rat Models. A Review of the Literature. Front. Cell. Neurosci 12: 1-20.

34. Sedaghati T, Jell G, Seifalian AM (2015) Nerve Regeneration and Bioengineering. Regenerative Medicine Applications in Organ Transplantation 31: 799-810.

35. Zhao Z, et al. (2014) Improvement in Nerve Regeneration through a Decellularized Nerve Graft by Supplementation with Bone Marrow Stromal Cells in Fibrin. Cell Transplant 23: 97-110

36. Guo Y, et al. (2013) Sensory recovery following decellularized nerve allograft transplantation for digital nerve repair. J. Plast. Surg. Hand Surg 47: 1-3.

37. Algarrahi K, et al. (2015) Acellular bi-layer silk fibroin scaffolds support functional tissue regeneration in a rat model of onlay esophagoplasty. Biomaterials 53: 149-159.

38. Kropp BP, et al. (1996) Characterization of Small Intestinal Submucosa Regenerated Canine Detrusor. J. Urol. 599-607.

39. Iop L, et al. (2014) Decellularized Allogeneic Heart Valves Demonstrate SelfRegeneration Potential after a Long-Term Preclinical Evaluation. PLoS One 9: e99593.
40. Boulze Pankert M, et al. (2014) Biocompatibility and Functionality of a TissueEngineered Living Corneal Stroma Transplanted in the Feline Eye. Invest. Ophthalmol. Vis. Sci. 55: 6908-6920.

41. Urciuolo A, et al. (2018) Decellularised skeletal muscles allow functional muscle regeneration by promoting host cell migration. Sci. Rep 8: 8398 .

42. Kasukonis B, et al. (2016) Codelivery of Infusion Decellularized Skeletal Muscle with Minced Muscle Autografts Improved Recovery from Volumetric Muscle Loss Injury in a Rat Model. Tissue Eng. Part A 22: 1151-1163.

43. Quarta M, et al. (2017) Bioengineered constructs combined with exercise enhance stem cell-mediated treatment of volumetric muscle loss. Nat Commun 8: 15613.

44. Trevisan C, et al. (2019) Allogenic tissue-specific decellularized scaffolds promote long-term muscle innervation and functional recovery in a surgical diaphragmatic hernia model. Acta Biomater 89: 115-125.

45. Zhu M, et al. (2019) In vivo engineered extracellular matrix scaffolds with instructive niches for oriented tissue regeneration. Nat. Commun 10: :4620.

46. Wang H, et al. (2015) Decellularization technology in CNS tissue repair. Expert Rev Neurother 15: 493-500.

47. DeQuach JA, et al. (2011) Decellularized Porcine Brain Matrix for Cell Culture and Tissue Engineering Scaffolds. Tissue Eng. Part A 17: 2583-2592.

48. Giobbe GG, et al. (2019) Extracellular matrix hydrogel derived from decellularized tissues enables endodermal organoid culture. Nat. Commun 10: 5658.

49. Costantini M, et al. (2017) Microfluidic-enhanced 3D bioprinting of aligned myoblastladen hydrogels leads to functionally organized myofibers in vitro and in vivo. Biomaterials 131: 98-110.

50. Capel AJ, et al. (2019) Scalable 3D printed molds for human tissue engineered skeletal muscle. Front. Bioeng. Biotechnol 7:20.

Copyright: (C2020 Raffa P. This is an open-access article distributed under the terms of the Creative Commons Attribution License, which permits unrestricted use, distribution, and reproduction in any medium, provided the original author and source are credited. 